\title{
Long optical plateau in the afterglow of the short GRB 150424A with extended emission
}

\section{Evidence for energy injection by a magnetar?}

\author{
F. Knust ${ }^{1}$, J. Greiner ${ }^{1}$, H. J. van Eerten ${ }^{2}$, P. Schady ${ }^{1}$, D. A. Kann ${ }^{3}$, T.-W. Chen ${ }^{1}$, C. Delvaux ${ }^{1}$, J. F. Graham ${ }^{1}$, \\ S. Klose ${ }^{4}$, T. Krühler ${ }^{1}$, N. J. McConnell ${ }^{5}$, A. Nicuesa Guelbenzu ${ }^{4}$, D. A. Perley ${ }^{6}$, S. Schmidl ${ }^{4}$, T. Schweyer ${ }^{1}$, \\ M. Tanga ${ }^{1}$, and K. Varela ${ }^{1}$
}

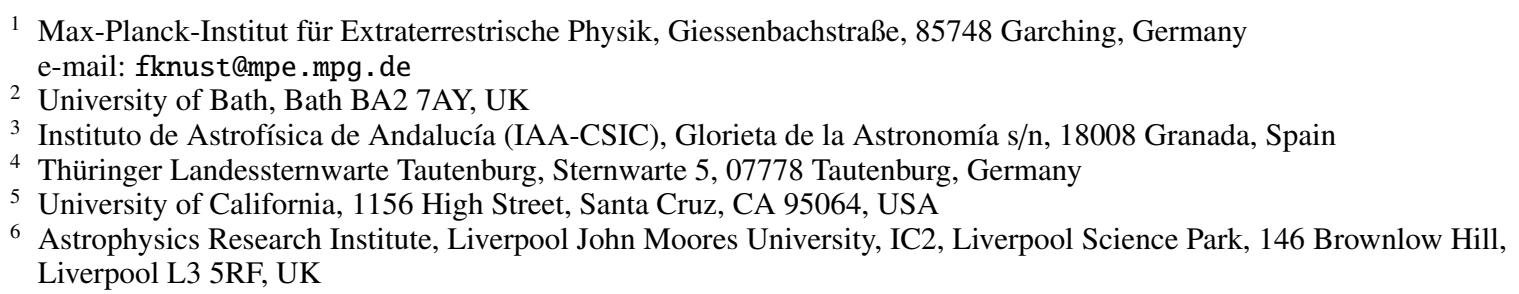

Received 8 February 2017 / Accepted 3 July 2017

\begin{abstract}
Context. Short-duration gamma-ray bursts (GRBs) with extended emission form a subclass of short GRBs, comprising about $15 \%$ of the short-duration sample. Afterglow detections of short GRBs are also rare (about 30\%) because of their lower luminosity.

Aims. We present a multiband data set of the short burst with extended emission, GRB 150424A, comprising of GROND observations, complemented with data from Swift/UVOT, Swift/XRT, HST, Keck/LRIS, and data points from the literature. The GRB 150424A afterglow shows an extended plateau phase, lasting about $8 \mathrm{~h}$. The analysis of this unique GRB afterglow might shed light on the understanding of afterglow plateau emission, the nature of which is still under debate.

Methods. We present a phenomenological analysis made by applying fireball closure relations and interpret the findings in the context of the fireball model. We discuss the plausibility of a magnetar as a central engine, which would be responsible for additional and prolonged energy injection into the fireball.

Results. We find convincing evidence for energy injection into the afterglow of GRB 150424A. We find that a magnetar spin-down as the source for a prolonged energy injection requires that at least $4 \%$ of the spin-down energy is converted into radiation.
\end{abstract}

Key words. gamma-ray burst: general - gamma-ray burst: individual: 150424A - methods: observational - methods: data analysis

\section{Introduction}

Gamma-ray bursts (GRBs) are among the most luminous explosions in the Universe. They are characterized by an intense prompt $\gamma$-ray flash, followed by a broadband afterglow. We distinguish between two flavors: short-duration GRBs and longduration GRBs (Kouveliotou et al. 1993). Some of the short GRBs show extended emission (EE) in the $\gamma$ band after the short $\gamma$ flash (e.g., Mazets et al. 2002; Norris \& Bonnell 2006; Norris et al. 2010). While classically GRBs that emit $90 \%$ of their prompt emission energy in $T_{90} \lesssim 2 \mathrm{~s}$ are classified as short, short GRBs with EE can have a significantly longer $T_{90}$. Since $\mathrm{EE}$ is also spectrally softer, the classification according to $T_{90}$ is debated.

Short GRBs are generally about a factor of 10-100 less energetic than the more common long GRBs (Ghirlanda et al. 2009), and they also have fainter afterglows (Berger 2007, 2010; Nakar 2007; Gehrels et al. 2008; Nysewander et al. 2009; Kann et al. 2011), which makes follow-up observations challenging.

Short GRBs are believed to originate from older stellar populations (for reviews, see, e.g., Fong et al. 2013; Berger 2014) and often occur at a relative offset to the center of the host galaxy
(Belczynski et al. 2006; Fong et al. 2010; Church et al. 2011; Fong \& Berger 2013; Behroozi et al. 2014). Afterglow analysis in the context of the "fireball model" (Mészáros \& Rees 1997; for reviews, see in e.g. Piran 2004; van Eerten 2015) implies that they have a relatively low circumburst density (median density $n \approx(3-15) \times 10^{-3} \mathrm{~cm}^{-3}, 80 \%-95 \%$ of the bursts have densities of $n \lesssim 1 \mathrm{~cm}^{-3}$, Fong et al. 2015).

The possible progenitors of short GRBs are still under some debate. The most favored progenitors today are compact binary mergers (CBMs). CBMs as progenitors are supported by evidence for a binary neutron star merger (kilonova) association (Tanvir et al. 2013; Berger 2014). The short GRB rate is also consistent with the expected CBM rate (Fong et al. 2012; Wanderman \& Piran 2015). The offsets of short GRBs to the center of their host galaxies correspond with the theoretical predictions for the kick a compact binary receives when formed (Berger 2010). Moreover, unlike for long GRBs, core-collapse supernova are ruled out because we lack observational associations (Hjorth et al. 2005a).

During the merging process, the two neutron stars can either directly collapse into a black hole, or form a strongly 


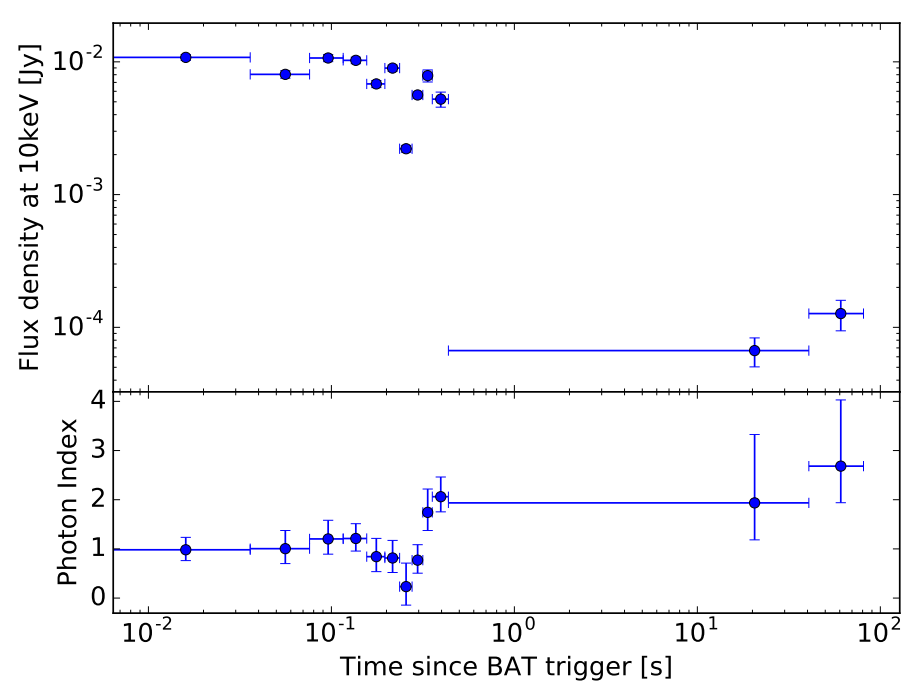

Fig. 1. Gamma-ray light curve of GRB 150424A. After the initial spike, the GRB shows extended emission for $\sim 100 \mathrm{~s}$ after the BAT trigger.

magnetized and rapidly rotating neutron star: a magnetar (Duncan \& Thompson 1996; Yi \& Blackman 1997; Metzger et al. 2008; Zhang \& Mészáros 2001. A magnetar would lose energy through dipole radiation and could provide a prolonged energy injection into the GRB blast wave. This energy injection would explain a "plateau phase". A plateau phase is a shallow decay phase in the afterglow light curve with a temporal slope $\alpha \lesssim 1 / 4^{1}$, where the standard model predicts a temporal slope $\alpha \sim 1$. However, the local magnetar rate does not agree with the GRB rate (Rea et al. 2015).

Since the first optical detection of an afterglow from a short GRB (Hjorth et al. 2005b), fewer than 90 short GRB $\mathrm{X}$-ray afterglows have been detected, as compared to around 1000 long GRB afterglows ${ }^{2}$. Only about $30 \%$ of all short GRB afterglows had an optical/near-infrared counterpart (Fong et al. 2015). Of these afterglows, only a few show a plateau phase: GRB 060313A (Roming et al. 2006), GRB 061201A (Stratta et al. 2007), and GRB 130603B (Fan et al. 2013; de Ugarte Postigo et al. 2014); see also Kann et al. (2011) for GRB 060313A, GRB 061201A, and GRB 090510A).

In this work we analyze afterglow data of GRB 150424A: an short GRB with EE and early multiband coverage. We use data from the Gamma-ray burst Optical Near-infrared Detector (GROND; Greiner et al. 2008), the Swift/Ultraviolet and Optical Telescope (UVOT; Roming et al. 2005), the Swift/X-Ray Telescope (XRT; Burrows et al. 2005), the Hubble Space Telescope (HST) and Keck/LRIS. This high-quality dataset makes GRB 150424A one of the best-detected short GRBs with EE and an optical plateau phase.

In Sect. 2 we present the data we used. In Sect. 3 we perform a phenomenological analysis and present its physical implications. In Sect. 4 we discuss the results and its implications for the physical nature of the GRB, followed by our conclusions in Sect. 5.

\section{Data}

On 24 April 2015 at 07:42:57 UT, the Swift Burst Alert Telescope (BAT) detected the short GRB150424A with a single

\footnotetext{
1 The sign of the slope is convention. We follow Eq. (1), where a positive number means a decaying light curve.

2 http://www.mpe.mpg.de/ jcg/grbgen.html
}

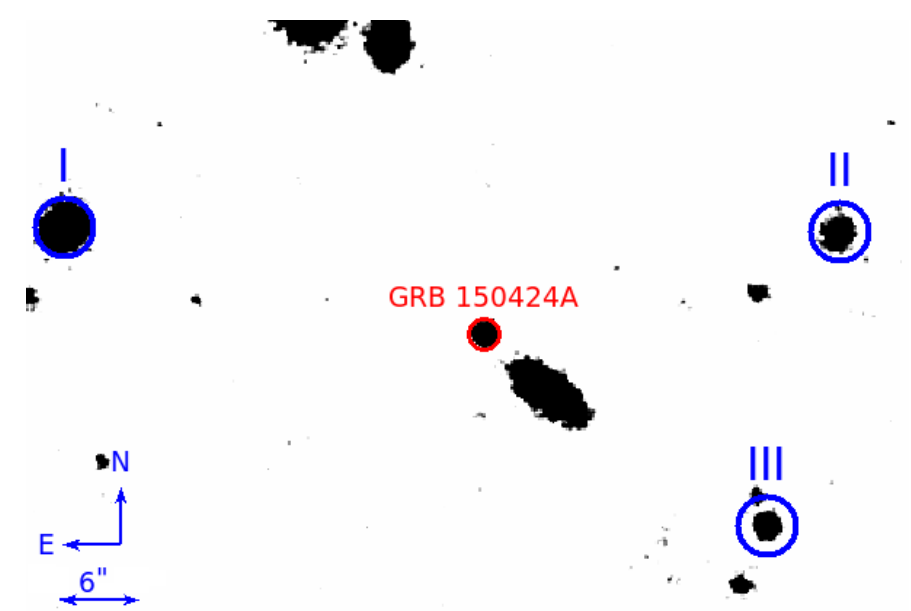

Fig. 2. GROND finding chart of GRB 150424A (GROND $r$ band). A galaxy lies southwest of the afterglow, but late-time HST observations find a weak expanded source at the position of the afterglow, which is believed to be the host.

peak of $0.5 \mathrm{~s}$ duration. Swift immediately slewed to the burst, and a fading X-ray counterpart was detected by the Swift/XRT (Beardmore et al. 2015). A non-fading optical counterpart was found (Marshall \& Beardmore 2015) with the Swift/UVOT (Roming et al. 2005). In a refined analysis, weak extended emission for $\sim 100 \mathrm{~s}$ was found (Barthelmy et al. 2015) and resulted in a $T_{90}=91 \pm 22 \mathrm{~s}$ ( the $\gamma$-ray light curve is shown in Fig. 1).

The UVOT observations cover the time from $82 \mathrm{~s}-1.4 \times$ $10^{6} \mathrm{~s}$ after the burst. UVOT photometry was carried out on pipeline-processed sky images downloaded from the Swift data center $^{3}$ following the standard UVOT procedure (Poole et al. 2008). Source photometric measurements were extracted from the UVOT early-time event data, and later we procured imaging data files using the tool UVOTMAGHIST (v1.1) with a circular source extraction region of $5^{\prime \prime}$ radius for the first $16 \mathrm{ks}$ of data, after which a $3.5^{\prime \prime}$ source region radius was used to maximize the signal-to-noise ratio. In order to remain compatible with the effective area calibrations, which are based on $5^{\prime \prime}$ aperture photometry, an aperture correction was applied on the photometry that was extracted using the smaller source aperture. We consider a signal-to-noise ratio of $\sigma>3$ as detection.

At $1.6 \mathrm{~h}$ after the burst, Perley \& McConnell (2015) reported a $g$ - and $R$-band detection of the afterglow with the Low Resolution Imaging Spectrometer (LRIS). GROND was able to follow up (Kann et al. 2015) $15 \mathrm{~h}$ after the burst. GROND detected the afterglow in the $g^{\prime}, r^{\prime}, i^{\prime}, z^{\prime}$ and $J$ band at RA, Dec $(J 2000)=$ 10:09:13.39, -26:37:51.46 (152.3058, -26.63096) with an uncertainty of $0.3^{\prime \prime}$, and observed strong fading at this point in time. All in all, GROND observations covered eight epochs with detections in the first three epochs. We performed aperture photometry on the LRIS and GROND data using our IRAF/PyRAF (Tody 1993) based pipeline (Yoldaş et al. 2008; Krühler et al. 2008), and calibrated the data against secondary field stars.

The GRB afterglow is northeast of an elliptical galaxy with a spectroscopic redshift $z=0.3$ (Castro-Tirado et al. 2015; see the finding chart in Fig. 2), but there are Hubble Space Telescope (HST) detections (Tanvir et al. 2015) of an extended source that is more likely to be the host galaxy at the position of the afterglow. More HST data of the WFC3 instrument were obtained

\footnotetext{
3 www.swift.ac.uk/swift_portal
} 


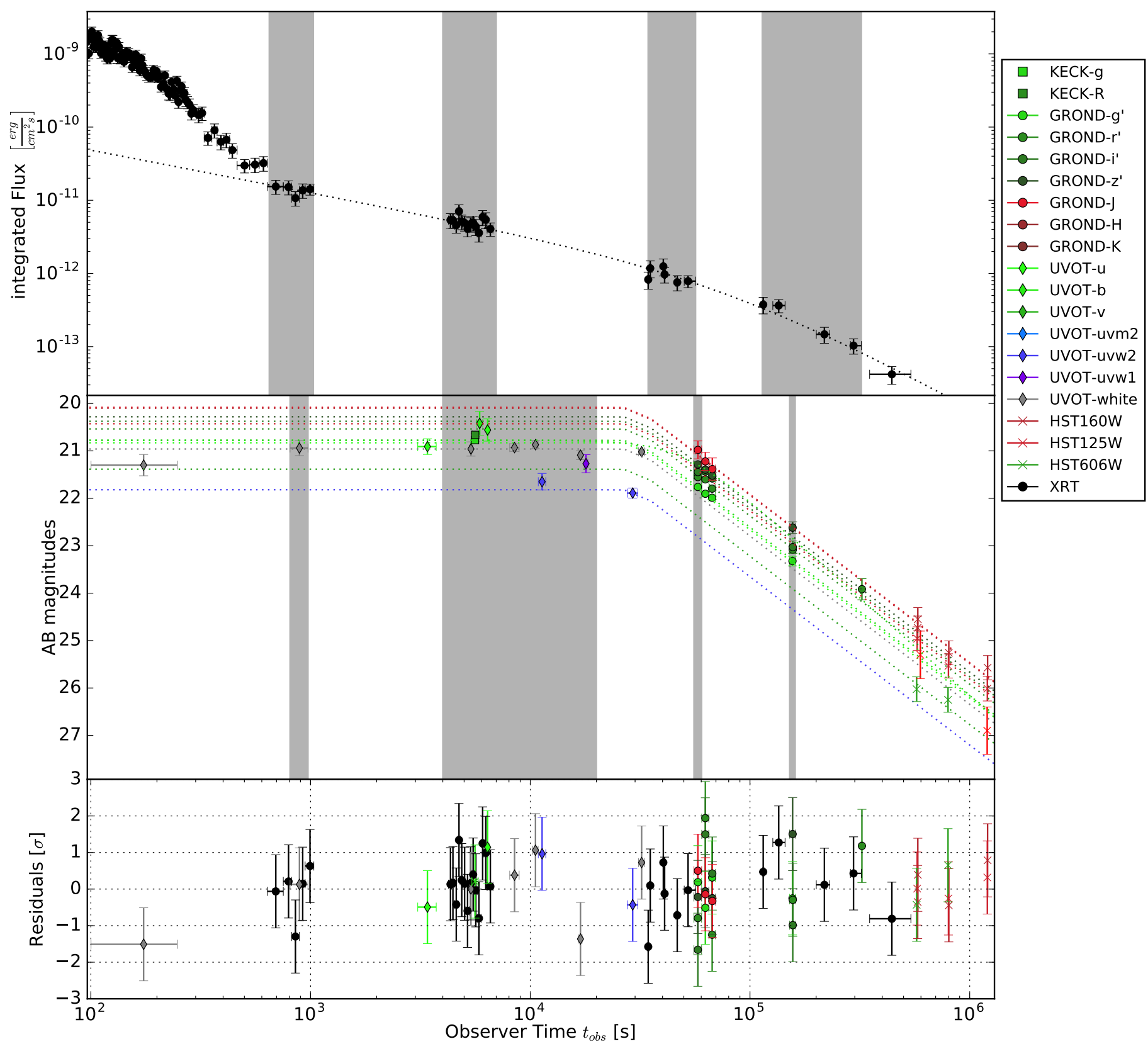

Fig. 3. XRT and GROND light curves of GRB 150424A. The shaded areas correspond to the time slices of the snapshot analysis. The dotted lines are the best-fitting smoothly broken power-laws. The X-ray light-curve fit has a reduced $\chi^{2}=0.57$, the optical light-curve fit has a reduced $\chi^{2}=1.04$. We fit all bands with more than one detection: $g^{\prime}, r^{\prime}, i^{\prime}, z^{\prime}, J$, white, $u, u v w 2$, HST160W, HST125W, and HST606W. All detections and upper limits (not shown in the light curve) are given in Table A.3. The temporal slopes $\alpha$ are given in Table 1.

from the data archive at the Space Telescope Science Institute ${ }^{4}$. We performed aperture photometry using a $0.4^{\prime \prime}$ aperture.

Fong (2015) reported a VLA 9.8 GHz detection $18 \mathrm{~h}$ after the trigger, and Kaplan et al. (2015) reported early MWA upper limits in the $\mathrm{MHz}$ regime.

\section{Analysis}

\subsection{Phenomenology}

A GRB afterglow can be described by the empirical flux description

$F_{v}(t) \propto t^{-\alpha} v^{-\beta}$

\footnotetext{
4 http://archive.stsci.edu/hst/index.html
}

with time $t$ and observed frequency $v$. The temporal slope $\alpha$ and the spectral slope $\beta$ depend on the observed spectral regime and can change over time (Mészáros \& Rees 1997; Granot \& Sari 2002).

The optical light curve of GRB 150424A (see Fig. 3) consists of two segments, a plateau and a decay phase. Both phases are covered by the UVOT observations. The GROND observations cover the decay phase and constrain its temporal slope. We fit all optical bands with more than one detection with one smoothly broken power-law per band. They all share their temporal slopes $\alpha$ and break time $t_{\text {break,opt }}$.

The X-ray light curve shows a steep decay until around $10^{3} \mathrm{~s}$, followed by a shallow decay phase, steepening again after $\sim 10^{5} \mathrm{~s}$. The steep decay phase is most likely due to the tail of prompt emission, so we ignore the $\mathrm{X}$-ray data before $10^{3} \mathrm{~s}$, since 
Table 1. Summary of the phenomenological analysis.

\begin{tabular}{c|cc|cc|cc|c}
\hline \hline \multirow{2}{*}{ GRB } & \multirow{2}{*}{$t_{\text {break,opt }}$} & \multirow{2}{*}{$t_{\text {break,xrt }}$} & \multicolumn{4}{|c}{ Optical } & \multicolumn{4}{c}{ X-rays } \\
& & & $\alpha$ & $\beta$ & $\alpha$ & $\beta$ & \\
\hline \multirow{2}{*}{$150424 \mathrm{~A}$} & \multirow{2}{*}{$30.4 \pm 0.9 \mathrm{ks}$} & $81.8 \pm 49.7 \mathrm{ks}$ & $0.00 \pm 0.01$ & $0.41_{-0.07}^{+0.11}$ & $0.58 \pm 0.07$ & $0.91_{-0.07}^{+0.11}$ & $t<t_{\text {break }}$ \\
& & & $1.42 \pm 0.05$ & $0.41_{-0.07}^{+0.11}$ & $1.66 \pm 0.26$ & $0.91_{-0.07}^{+0.11}$ & $t>t_{\text {break }}$ \\
\hline & & & \multicolumn{2}{|c|}{ smoothness: $15.0 \pm 13.8$} & smoothness: $1.0 \pm 0.8$ & \\
\hline
\end{tabular}

Notes. Temporal slopes $\alpha$ and spectral slopes $\beta$ as defined in Eq. (1).

Table 2. Additional data for the GRB.

\begin{tabular}{c|cl}
\hline \hline & $150424 \mathrm{~A}$ & Reference \\
\hline Galactic $E(B-V)[\mathrm{mag}]$ & $0.051 \pm 0.002$ & Schlafly \& Finkbeiner (2011) \\
Galactic $N_{\mathrm{H}}\left[10^{21} \mathrm{~cm}^{-2}\right]$ & 0.60 & Kalberla et al. (2005) \\
$z$ & $1.0_{-0.2}^{+0.3}$ & afterglow photometry \\
$d_{L}\left[10^{28} \mathrm{~cm}\right]$ & 2.1 & from $z$ \\
Host $E(B-V)[\mathrm{mag}]$ & $0.0_{-0.0}^{+1.5}$ & SED fit \\
Host $N_{\mathrm{H}}\left[10^{22} \mathrm{~cm}^{-2}\right]$ & $0.04_{-0.04}^{+0.24}$ & SED fit \\
$R_{V}$ & $3.08(\mathrm{MW})$ & assumption \\
\hline
\end{tabular}

a standard fireball afterglow model is not applicable. We fit the remaining the X-ray light curve with a smoothly broken power law.

To determine the spectral slopes $\beta$, we performed a joined broadband fit of optical and X-ray data using XSPEC (Arnaud 1996). The fireball model assumes synchrotron as the underlying emission mechanism, therefore the spectral slopes only depend on the electron energy distribution. The model uses a powerlaw-like electron energy distribution with slope $p$. Assuming a constant $p$, the spectral slopes do not change. The light-curve fit supports the assumption, since thanks to the multiband capabilities of GROND, a chromatic break would be clearly seen in the residuals around the optical break time.

We chose four time slices over which we integrated the XRT spectral counts (indicated with the gray bars in Fig. 3). Then we rebinned each spectrum, where we had to find an optimum between counts per spectral bin and number of spectral bins. For SED2 and SED3 we renormalized the total flux of the spectrum to the flux of the XRT light curve at the point in time of the optical data. For SED0 and SED1 we do not expect the total flux to be significantly different from the flux at the point in time of the optical data. Afterwards, we add a systematic error of $10 \%$ to account for the flux calibration relative to the optical data.

First, we corrected the optical/NIR and UV data for galactic extinction $E(B-V)=0.0513 \pm 0.0024$ (Schlafly \& Finkbeiner 2011), then we used a model consisting of a (broken) power law, and involving galactic absorption $N_{\mathrm{H}}=0.602 \times 10^{21} \mathrm{~cm}^{-2}$ (Kalberla et al. 2005), and host extinction and absorption. We fit the four SEDs simultaneously, while each single SED had an individual break frequency and normalization. We found the spectral slopes before and after the temporal break to be consistent, so for the final fit, we linked them for all four SEDs. We also used the constraint $\beta_{\text {optical }}-\beta_{\mathrm{X} \text {-ray }}=0.5$ for a synchrotron SED in the slow-cooling case with the cooling frequency $v_{\mathrm{c}}$ between optical and X-ray. When we do not set this constraint, the spectral slopes and the break frequency are degenerate. The resulting optical slope is also consistent with the fast-cooling case, where $\beta_{\mathrm{opt}}=0.5$.

The SED fits including the data and the unfolded model (only the power law, without extinction and absorption) are shown in Fig. 4. All results of our analysis for $\alpha$ and $\beta$ are given in Table 1 . A summary of the physical values from the spectral fitting is listed in Table 2.

The radio detection from Fong (2015) at time $t_{\mathrm{SED} 2}=$ 57900 s allows us to constrain the peak of the SED and the two characteristic frequencies (see Fig. 5). For the spectral slope below the maximum frequency we assume the standard fireball $\beta=-1 / 3$, the other spectral slopes are those derived in the multiSED fit.

Figure 6 shows the evolution of the characteristic frequencies based on our four SEDs, and the evolution of the characteristic frequencies according to some physical models, which we discuss in Sect. 4.

\subsection{Closure relations}

Within the fireball model, a GRB afterglow is explained as synchrotron radiation of shock-accelerated electrons from an ultra-relativistic outflow hitting the circumburst medium. In a dynamical afterglow model, the temporal and spectral behavior of an afterglow can be linked over a set of so-called closure relations between $\alpha$ and $\beta$. They depend on the state and structure (isotropic or jet) of the outflow, the circumburst density profile, and the energy distribution of the electrons.

The dynamic of the relativistic outflow is influenced by the circumburst medium. For long GRBs, two scenarios are usually considered. First, the interstellar medium (ISM) case, where the circumburst medium is assumed to be homogeneous. Second, the stellar wind case, where the circumburst medium has a radial density profile $n=n_{0}\left(R / R_{0}\right)^{-2}$, with a reference density $n_{0}$ and a reference radius $R_{0}$. For short GRBs the ISM case is expected. 


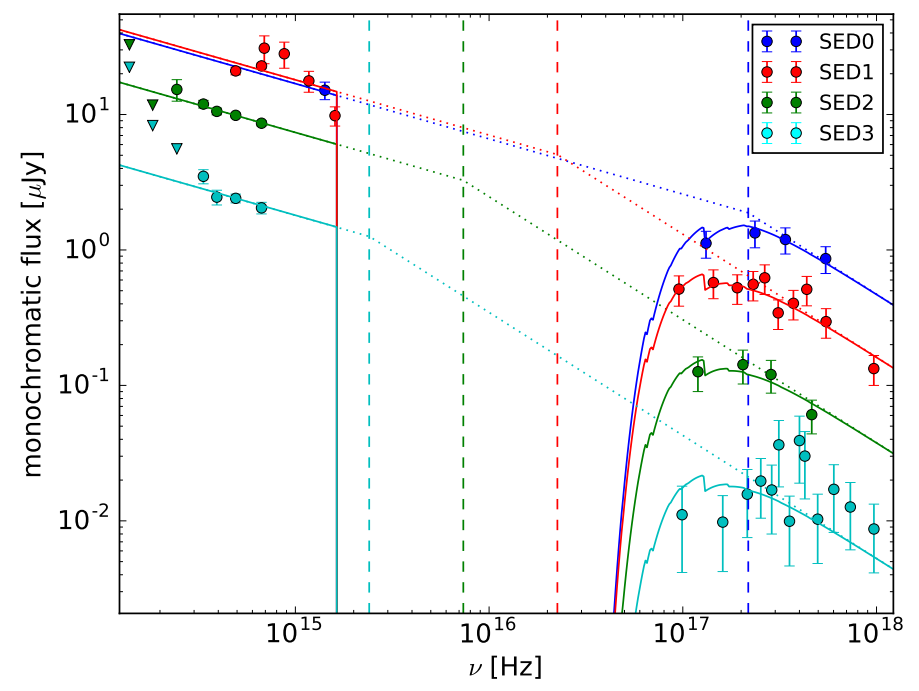

Fig. 4. Broadband fit of four SEDs during the time bins indicated in Fig. 3. All four SEDs share the same spectral slopes. The solid lines are the folded model, and the dotted lines are the unfolded model. The vertical dashed lines mark the characteristic break frequency. A characteristic frequency clearly evolves toward the low-energy part of the spectrum.

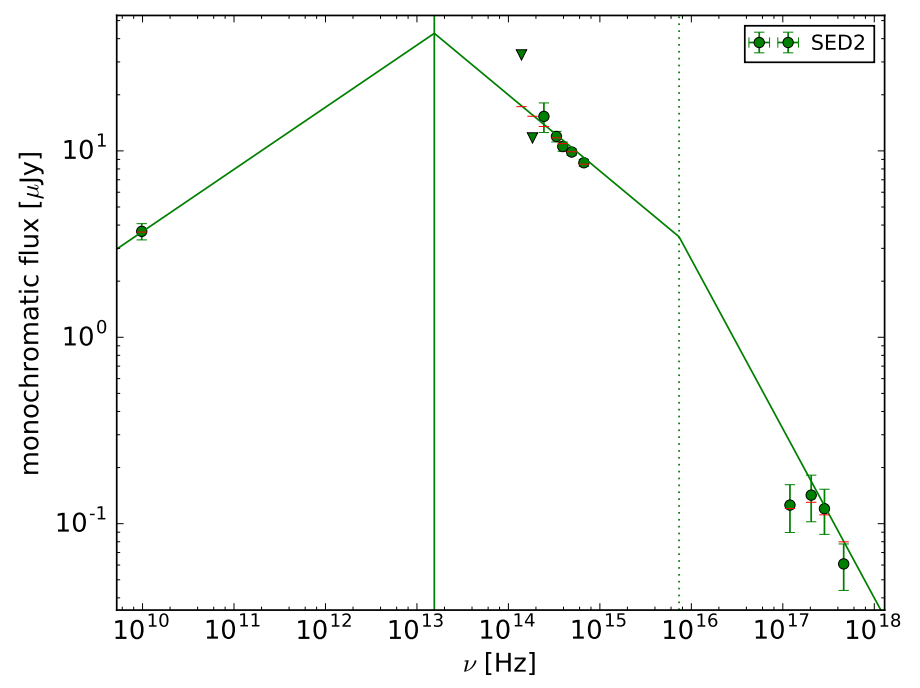

Fig. 5. Unfolded SED $2 t_{\mathrm{SED} 2}=57900 \mathrm{~s}$ after the trigger. The radio detection allows us to determine the peak frequency of the spectrum, using a spectral slope $\beta=-1 / 3$ below the peak frequency, and the spectral slope derived in the multi-SED fit.

They are thought to be the result of a CBM, and a compact binary system does not produce a stellar wind during its lifetime.

A synchrotron spectrum is conveniently characterized by characteristic frequencies: the injection frequency $v_{m}$, which derives from the peak of the electron energy distribution, and the cooling frequency $v_{\mathrm{c}}$, above which the electrons lose a significant amount of energy via synchrotron cooling. The slowcooling case is defined as $v_{m}<v_{\mathrm{c}}$, and the fast-cooling case is defined as $v_{m}>v_{\mathrm{c}}$.

We compared the fitted $\alpha$ and $\beta$ to the theoretical closure relations collected by Racusin et al. (2009; from Zhang \& Mészáros 2004; Zhang et al. 2006; Dai \& Cheng 2001; Panaitescu et al. 2006; Panaitescu 2005). We abbreviate the closure relations with CR 1-14 (see Table 1 in Racusin et al. 2009, and Table A.1). We adopted the following scheme: we used the $\beta$ value fitted to the data. Then we calculated $p(\beta)$. Racusin et al. (2009) described

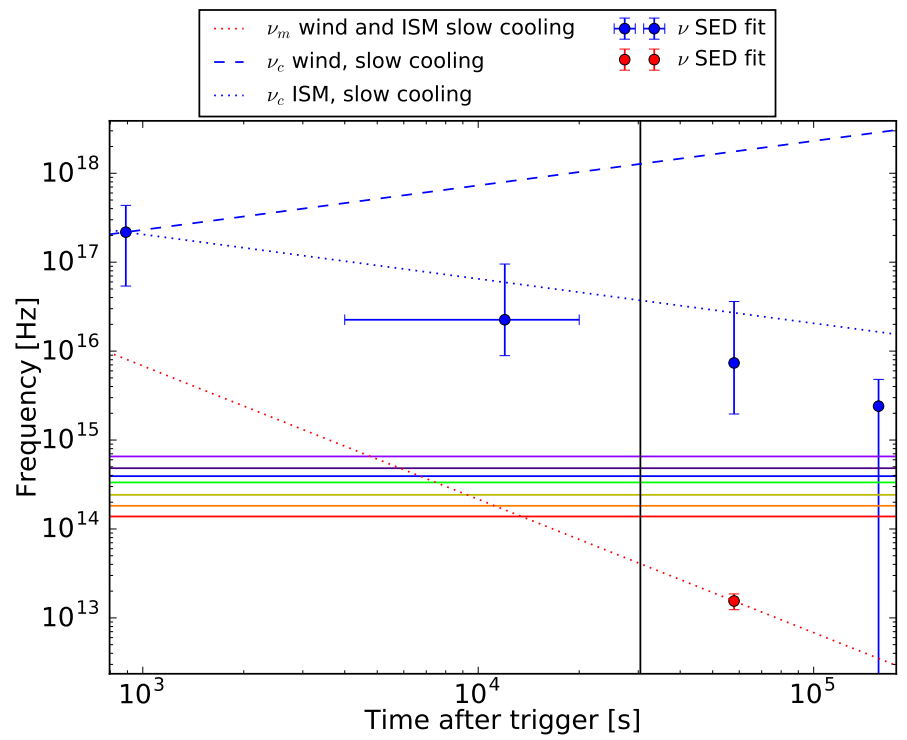

Fig. 6. Evolution of the characteristic frequencies from the SED fit and the standard fireball model. The horizontal lines are the GROND bands. The vertical black line corresponds to the optical break time. The blue data points are the break frequencies of the multi-SED fit. The red data point is the peak frequency of the fit of SED2. The dashed and dotted lines are the evolutions of the characteristic frequencies according to different fireball scenarios.

multiple ways to calculate $\alpha$. They differ if $p>2$ or $p<2$, and if we wish to consider energy injection. We first calculated $\alpha(\beta)$ without energy injection. If the calculated $\alpha$ was consistent with the $\alpha$ we fitted to the data, we considered it a "plausible scenario", if it was not, we calculated the energy injection index $q(\alpha, \beta) . q$ is defined using

$L_{\text {inj }}(t)=L_{0}\left(t / t_{b}\right)^{-q}$

and is valid for $q \leqslant 1$. $L_{\text {inj }}$ is the luminosity injected into the blast wave. The time $t$ and break time $t_{b}$ are given in the observer frame. $q=1$ is the impulsive injection case, and $q=0$ corresponds to a constant energy injection from a magnetar spindown, for instance (Zhang et al. 2006). We checked all $\alpha-\beta$ pairs from the phenomenological analysis (Table 1). In Table A.1 we list all closure relations that describe our afterglow, and the $q$ values if needed.

In the SED fits we saw a break between the spectral regimes, so a set of closure relations can only be consistent with both spectral regimes if we find closure relations that lie on different sides of a characteristic frequency, and describe the same scenario. After the break, we only consider scenarios without energy injection to be plausible.

We find only one scenario that describes both spectral regimes (optical and X-ray), before and after the break: a uniform non-spreading jet in an ISM environment. After the temporal break, the scenario is consistent with the optical data (CR11, $v_{m}<v<v_{\mathrm{c}}$ ) and the X-ray (CR12, $v>v_{\mathrm{c}}$ ), without the need for energy injection. Before the temporal break, the optical data imply the need for energy injection with an injection index $q_{\mathrm{opt}}=0.06 \pm 0.07$, and the X-ray data imply a consistent $q_{x}=0.14 \pm 0.11$.

\subsection{Redshift and host}

Using the extinction-corrected SED1 (see Fig. 7), we obtain the redshift from the afterglow as described by Krühler et al. (2011), 


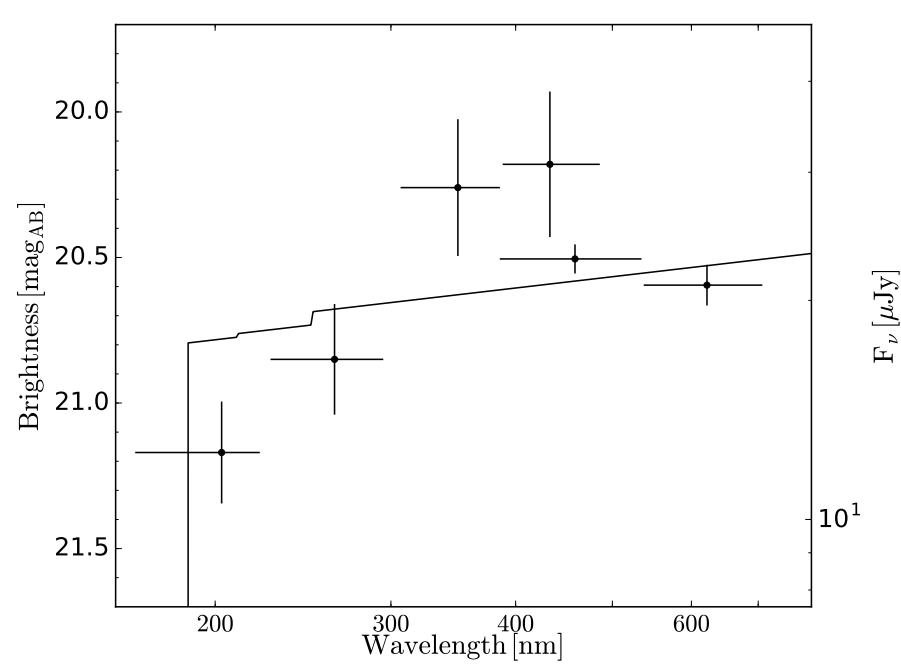

Fig. 7. Best-fitting power-law template for the afterglow SED1. A $z=$ $1.0_{-0.2}^{+0.3}$ is implied.

and find $z=1.0_{-0.2}^{+0.3}$. In a standard $\Lambda$ CDM cosmology with $H_{0}=$ $67.3 \frac{\mathrm{km} \mathrm{s}^{-1}}{\mathrm{Mpc}}, \Omega_{\mathrm{m}}=0.315, \Omega_{\Lambda}=0.685$ (Planck Collaboration XVI 2014), this corresponds to a distance modulus of 44.2 mag and a luminosity distance of $D_{L}=2.1 \times 10^{28} \mathrm{~cm}$.

We note that both the size $\left(<1^{\prime \prime}\right.$, corresponding to $<8 \mathrm{kpc}$ diameter) and the absolute luminosity $\left(M_{B} \sim-17.2 \pm 0.5 \mathrm{mag}\right)$ of the host are unusual for short-duration GRBs.

\subsection{Plausibility of a magnetar central engine}

Zhang \& Mészáros (2001) suggested a magnetar model as short GRB progenitor with a prolonged energy injection. According to this model, the magnetic field strength $B_{0 p}$ at the poles of a magnetar is linked to its spin-down luminosity $L_{\mathrm{sd}}$, and the initial spin period $P_{0}$ of the magnetar is linked to the spin-down time of the magnetar $\tau$ :

$B_{0 p, 15}^{2}=4.20 I_{45}^{2} R_{6}^{-6} L_{\mathrm{sd}, 49}^{-1} \tau_{\mathrm{sd}, 3}^{-2}$

$P_{0,-3}^{2}=2.05 I_{45} L_{\mathrm{sd}, 49}^{-1} \tau_{\mathrm{sd}, 3}^{-1}$,

with the moment of inertia $I_{45}$ in $10^{45} \mathrm{~g} \mathrm{~cm}^{2}$. The spin-down luminosity of the magnetar $L_{\mathrm{sd}, 49}$ is given in $10^{49} \mathrm{erg} / \mathrm{s} . B_{0 p, 15}$ is in units of $10^{15}$ Gauss. In the special case of a short GRB with EE, $P_{0}$ corresponds to the spin period after the EE (Zhang et al. 2007; Gompertz et al. 2013), rather than the spin-down period when formed. $\tau_{\mathrm{sd}, 3}$ is the spin-down energy release timescale in units of $10^{3} \mathrm{~s}$, which corresponds to our plateau end time, respectively, the break time $t_{\text {break,xrt }}$. Following Zhang \& Mészáros (2001), we adopted a radius of the neutron star $R_{6}=10^{6} \mathrm{~cm}$ and a neutron star mass $m=1.4 M_{\odot}$, which leads to $I=$ $1.85 \times 10^{45} \mathrm{~g} \mathrm{~cm}^{2}$.

The efficiency with which $L_{\mathrm{sd}, 49}$ is converted into the afterglow luminosity in a specific band $X$ is

$L_{\mathrm{X}} \equiv \eta_{1} L_{\mathrm{BOL}} \equiv \eta_{1} \eta_{2} L_{\mathrm{inj}} \equiv \eta_{12} L_{\mathrm{inj}} \equiv \eta_{12} L_{\mathrm{sd}}$,

with the bolometric afterglow luminosity $L_{\mathrm{BOL}}$, the luminosity injected into the blast wave $L_{\mathrm{inj}}$, the observed luminosity $L_{\mathrm{X}}$, and the corresponding efficiency factors $\eta$. We assumed that all the magnetar spin-down luminosity is injected into the blast wave $L_{\text {inj }}=L_{\text {sd }}$.
Generally, $\eta_{12}$ is time dependent, since the characteristic quantities of a synchrotron spectrum in the fireball model also evolve with time. For a constant energy injection $q=0$ and a $p \sim 2$, this time dependence is expected to be weak, in an ISM environment $k=0$, or in a wind environment $k=2$ (see the fireball flux equation in, e.g., van Eerten 2014).

We used the break time $t_{\text {break,xrt }}$ of the X-ray light curve and SED2 to calculate the rest frame luminosity at that time. Gompertz et al. (2013), Rowlinson et al. (2014), Rea et al. (2015) approximated the bolometric luminosity with the 1$10000 \mathrm{keV}$ band, extrapolated from Swift data. When we used the lumin command of Xspec and a dummy response, it led to a luminosity $L_{1-10000 \mathrm{keV}}=2.6 \times 10^{46} \mathrm{erg} \mathrm{s}^{-1}$. When we integrated SED2 $\left(10^{-14}-10^{4} \mathrm{keV}\right)$, we found a similar $L_{0-10000 \mathrm{keV}}=2.7 \times$ $10^{46} \mathrm{erg} \mathrm{s}^{-1}$. Assuming a $10 \%$ error in the luminosity and $\eta_{12}=$ 1 , this results in magnetar parameters $B_{0 p}=(0.9 \pm 0.5) \times 10^{15} \mathrm{G}$ and $P_{0}=4.3 \pm 1.3 \mathrm{~ms}$.

Since naturally $\eta_{12}<1$, our values for $B_{0 p}$ and $P_{0}$ therefore have to be seen as upper limits. Moreover, a $\eta_{12}=1$ would mean that all the injected luminosity is radiated away immediately, and nothing goes into the kinetic energy of the outflow. The fireball model can therefore only be self-consistent when $\eta_{12} \ll 1$.

Our $P_{0}$ lies above the mass-shedding limit $P_{0}=0.81 \mathrm{~ms}$ (Lattimer \& Prakash 2004) below which a neutron star would be disrupted by centrifugal forces. For our fitted $t_{\text {break,xrt }}$, the massshedding limit is reached when the $L_{\mathrm{sd}, \max }>(7 \pm 4) \times 10^{47} \mathrm{erg} \mathrm{s}^{-1}$. This yields an $\eta_{12} \gtrsim 4 \%$.

The isotropic energy in the $\gamma$ band is

$E_{\gamma, \text { iso }}=f_{\gamma} \times D_{L}(z)^{2} 4 \pi \times(1+z)^{-1}$,

with the $\gamma$-fluence $f_{\gamma}=15(1) \times 10^{-7} \mathrm{erg} \mathrm{cm}^{-2}$ measured by the $S$ wift $/ \mathrm{BAT}^{5}$ and the luminosity distance $D_{\mathrm{L}}$ follows $E_{\gamma \text {,iso }}=$ $4.1 \times 10^{51} \mathrm{erg}$. $E_{\gamma \text {,iso }}$ is a proxy for the impulsive energy input into the the blast wave (Granot et al. 2006). The prolonged energy injected is the luminosity at the end of the plateau times the length of the plateau. Assuming an $\eta_{12}=1$, the sum of both is the total energy of the blast wave $E_{\text {tot }}=E_{\gamma, \text { iso }}+t_{\text {break, } x r t} \times L_{0-10000 \mathrm{keV}}=$ $(6.3 \pm 1.4) \times 10^{51} \mathrm{erg}$ and does not exceed the limits for the maximum rotational energy of a proto-magnetar of $1-2 \times 10^{53} \mathrm{erg}$ suggested by Metzger et al. (2015).

\section{Discussion}

\subsection{General description}

The X-ray light curve of GRB 150424A shows a steep decay from the prompt emission, followed by a smoothly broken power-law. GRB 150424A seems to be a typical short GRB with EE (Gompertz et al. 2013). This GRB becomes special since it is one of the rare cases with an early multi-epoch optical coverage, during which the optical emission is basically constant for $8 \mathrm{~h}$. While the temporal break in the optical is very sharp, the break of the X-ray light curve is smoother. The breaks occur around the same time, however, which is a strong indicator that the underlying dynamics change at that point in time, that is, at the end of the optical plateau.

Studies concerning the relation between the end time of a plateau and the luminosity at the end of the plateau (Dainotti et al. 2008, 2013; Li et al. 2012) have shown that the afterglow of GRB 150424A represents a "typical" shallow-decay afterglow. As seen in Fig. 8, the afterglow does not have an

\footnotetext{
5 http://swift.gsfc.nasa.gov/archive/grb_table.html
} 


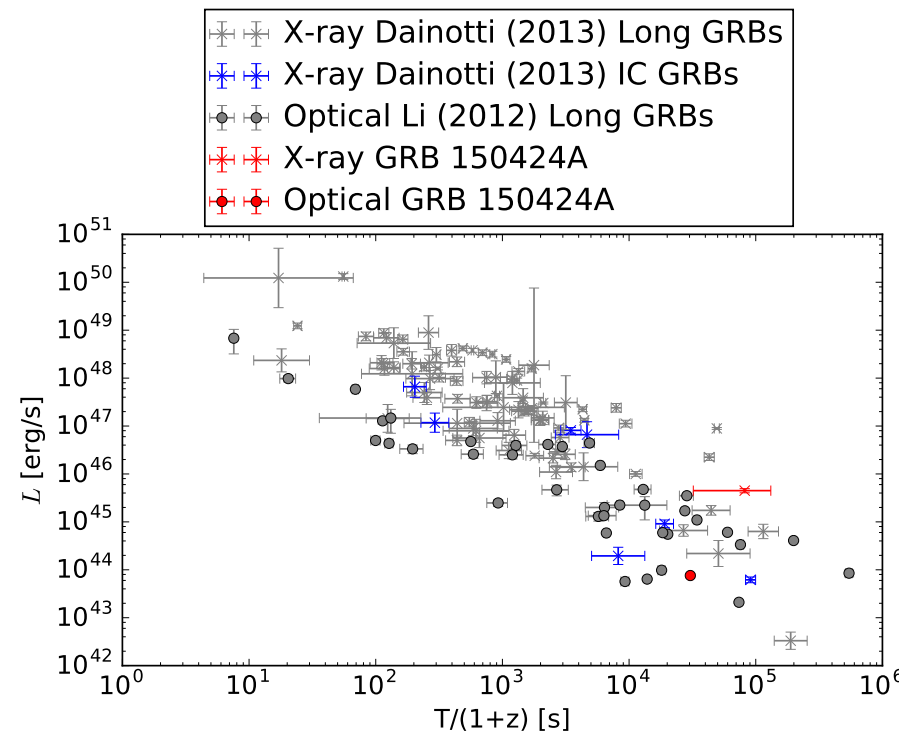

Fig. 8. Plateau end-time luminosity correlations. The X-ray data for long GRBs and short GRBs with EE (tagged as intermediate class (IC) GRBs) is taken from Dainotti et al. (2013; gray and blue crosses). The optical data are taken from Li et al. (2012; gray circles). The afterglow of GRB 150424A does not show any special behavior in the optical (red circle) or in X-rays (red cross).

outstanding position in the plateau end time - luminosity parameter space. The position of GRB 150424A in the parameter space is consistent with long GBRs, for the optical and Xray data. Comparing the position to other short GRBs, the afterglow has a slightly higher X-ray luminosity. However, the short GRB sample lacks optical data and is too small to conclude that GRB $150424 \mathrm{~A}$ is an atypical short GRB.

\subsection{Physical interpretation}

With an electron distribution index of $\sim 2$, an optical spectral slope of $\sim 1 / 2$, and an X-ray spectral slope of $\sim 1$, the spectral fits do not allow us to distinguish between a slow- or fast-cooling case when we use the spectral shapes given by Sari et al. (1998).

In Sect. 3.2 we tested the derived spectral and temporal slopes with the most common closure relations (see Table A.1). Here we present our findings. In Sects. 4.3 and 4.4 we discuss two standard afterglow scenarios and in Sect. 4.5 a scenario that involves energy injection as well.

\subsection{Scenario 1: ISM, slow cooling}

The spectral shape of SED2 is compatible with slow cooling in an ISM, and would allow us to constrain $v_{m}$ (see Fig. 5). The temporal evolution of $v_{\mathrm{c}}$ also fits the observation (blue dotted line and blue and red dots in Fig. 6).

The spectral shape from our SED fits shows that the X-ray data lie well above the cooling break. However, the X-ray data by themselves can only be explained by closure relations for slow cooling in an ISM if the observed X-ray frequency were $v_{m}<$ $v_{\mathrm{X} \text {-ray }}<v_{\mathrm{c}}(\mathrm{CR} 1)$, with energy injection before the break and no energy injection after the break. The SED fits and the closure relation CR1 for the X-ray light-curve therefore contradict each other.

\subsection{Scenario 2: slow cooling in wind without energy injection}

Granot \& Sari (2002) gave a temporal slope $\alpha=0$ for this scenario when the observer frequency is $v<v_{m}$. Therefore, the optical plateau could be explained if $v_{m}$ were higher than the optical frequencies. When we extrapolate from SED2 and follow the temporal evolution $v_{m} \propto t^{-3 / 2}$, we find that it would cross our observed bands at the time of SED1 (red dotted line in Fig. 6), before the temporal break in the optical. In our SED fits, SED0 has only one UVOT white-band data point, and for SED1 we had to use a wide time bin for the optical part of the SED. A $v_{m}$ crossing at that point in time may therefore not be detectable by our spectral fitting, but it should coincide with the end of the plateau.

Before the break, the X-ray data are not consistent with this scenario (to fulfill CR5 or CR6 in Table A.1, energy injection has to be accounted for). After the break, it is consistent when $v_{m}<v_{\mathrm{X} \text {-ray }}<v_{\mathrm{c}}(\mathrm{CR} 5)$. However, our SED fit shows that the fitted $v_{\mathrm{c}}$ is well below the X-ray (blue dots in Fig. 6) and that the evolution of the break frequency does not follow the predicted evolution for $v_{\mathrm{c}}$ in this scenario (see the blue dashed line and blue data points in Fig. 6).

\subsection{Scenario 3: uniform nonspreading jet in ISM with energy injection}

The closure relations for a uniform nonspreading jet in an ISM medium with slow cooling are valid for all temporal and spectral regimes (the optical is consistent with CR11, the X-ray is consistent with CR12). Before the temporal break, energy injection is needed, after the temporal break, it is consistent with both spectral regimes without energy injection. We derived an efficiency to convert the spin-down luminosity of the magnetar into the total afterglow luminosity of $\eta_{12} \gtrsim 4 \%$. Models that assume an adiabatic blast wave, like the one in we used in scenario 3, harmonize with a $4 \%$ loss that is due to radiation.

We derived the energy injection index $q$ independently from the optical and the X-ray data. Both $q$ values are consistent with a constant energy injection $(q=0)$, as expected from a magnetar.

In scenario 3 , the jet nature of the outflow is apparent immediately from the beginning of the light curve, which means that the jet break has already occurred before the observations began, before the first optical data point $\lesssim 200$ s. This is more than one order of magnitude earlier than the earliest short GRB jet break measurement (GRB 090426A, 35000 s, Nicuesa Guelbenzu et al. 2011), although it is debated whether GRB 090426A truly is a short GRB), or long GRB measurement (GRB 120729A 9500 s, Cano et al. 2014), and would imply a very narrow jet opening angle. The narrower the jet opening angle, the lower the beaming-corrected total energy output, given an isotropic energy equivalent.

\section{Conclusions}

We presented multiband data with a uniquely high temporal and spectral coverage of the short GRB 150424A with EE. We performed a phenomenological analysis and interpreted it in the context of the fireball model. We found three scenarios that explain parts of the temporal and spectral behavior of the afterglow: 1. Slow cooling in an ISM, 2. slow cooling in wind without energy injection, and 3. a uniform nonspreading jet in an ISM medium with energy injection. We found that typical standard scenarios of GRB afterglows, that is, slow cooling in ISM or wind environment, are not able to explain our data. 
In contrast, a uniform nonspreading jet expanding into an ISM medium that is repowered for $\approx 10^{4} \mathrm{~s}$ with additional constant energy injection can explain the data self-consistently. For a magnetar as supplier of this prolonged energy injection, an efficiency $\eta_{12} \gtrsim 4 \%$ in converting the spin-down luminosity of the magnetar $L_{\mathrm{sd}}$ into the afterglow luminosity has to be assumed.

The unique and very-long-duration energy injection provides, within a factor of 2 , a similar energy input into the surroundings as the prompt GRB emission. That the jet behavior is apparent from very early times on, however, implies an extremely narrow jet opening angle. The narrower the jet opening angle, the smaller the beaming-corrected total energy output. Even the total isotropic equivalent energy release is a factor of 20 below the maximum possible for a magnetar, however.

Acknowledgements. H.J.v.E. was supported by the Alexander von Humboldt foundation at the time of this work. D.A.K. acknowledges financial support from MPE, from TLS, from the Spanish research project AYA 2014-58381-P, and from Juan de la Cierva Incorporación fellowships IJCI-2015-26153 and IJCI2014-21669. P.S., T.W.C., J.F.G., M.T. acknowledge support through the Sofja Kovalevskaja Award from the Alexander von Humboldt Foundation of Germany. S.K. and A.N.G. acknowledge support by DFG grant K1 766/16-1. S.S. acknowledges support by the Thüringer Ministerium für Bildung, Wissenschaft und Kultur under FKZ 12010-514. Part of the funding for GROND (both hardware as well as personnel) was generously granted from the Leibniz-Prize to G. Hasinger (DFG grant HA 1850/28-1). This work made use of data supplied by the UK Swift Science Data Centre at the University of Leicester. Based on observations made with the NASA/ESA Hubble Space Telescope, obtained from the Data Archive at the Space Telescope Science Institute, which is operated by the Association of Universities for Research in Astronomy, Inc., under NASA contract NAS 5-26555. These observations are associated with program \# 13830 Pi'ed by N. Tanvir.

\section{References}

Arnaud, K. A. 1996, in Astronomical Data Analysis Software and Systems V, eds. G. H. Jacoby, \& J. Barnes, ASP Conf. Ser., 101, 17

Barthelmy, S. D., Baumgartner, W. H., Beardmore, A. P., et al. 2015, GRB Coordinates Network, 17761, 1

Beardmore, A. P., Page, K. L., Palmer, D. M., \& Ukwatta, T. N. 2015, GRB Coordinates Network, 17743, 1

Behroozi, P. S., Ramirez-Ruiz, E., \& Fryer, C. L. 2014, ApJ, 792, 123

Belczynski, K., Perna, R., Bulik, T., et al. 2006, ApJ, 648, 1110

Berger, E. 2007, ApJ, 670, 1254

Berger, E. 2010, ApJ, 722, 1946

Berger, E. 2014, ARA\&A, 52, 43

Burrows, D. N., Hill, J. E., Nousek, J. A., et al. 2005, Space Sci. Rev., 120, 165

Cano, Z., de Ugarte Postigo, A., Pozanenko, A., et al. 2014, A\&A, 568, A19

Castro-Tirado, A. J., Sanchez-Ramirez, R., Lombardi, G., \& Rivero, M. A. 2015, GRB Coordinates Network, 17758, 1

Church, R. P., Levan, A. J., Davies, M. B., \& Tanvir, N. 2011, MNRAS, 413, 2004

Dai, Z. G., \& Cheng, K. S. 2001, ApJ, 558, L109

Dainotti, M. G., Cardone, V. F., \& Capozziello, S. 2008, MNRAS, 391, L79

Dainotti, M. G., Petrosian, V., Singal, J., \& Ostrowski, M. 2013, ApJ, 774, 157

de Ugarte Postigo, A., Thöne, C. C., Rowlinson, A., et al. 2014, A\&A, 563, A62

Duncan, R. C., \& Thompson, C. 1996, in High Velocity Neutron Stars, eds. R. E. Rothschild, \& R. E. Lingenfelter, AIP Conf. Ser., 366, 111

Fan, Y.-Z., Yu, Y.-W., Xu, D., et al. 2013, ApJ, 779, L25

Fong, W. 2015, GRB Coordinates Network, 17804, 1

Fong, W., \& Berger, E. 2013, ApJ, 776, 18

Fong, W., Berger, E., \& Fox, D. B. 2010, ApJ, 708, 9
Fong, W., Berger, E., Margutti, R., et al. 2012, ApJ, 756, 189 Fong, W., Berger, E., Chornock, R., et al. 2013, ApJ, 769, 56 Fong, W., Berger, E., Margutti, R., \& Zauderer, B. A. 2015, ApJ, 815, 102 Gehrels, N., Barthelmy, S. D., Burrows, D. N., et al. 2008, ApJ, 689, 1161 Ghirlanda, G., Nava, L., Ghisellini, G., Celotti, A., \& Firmani, C. 2009, A\&A, 496, 585

Gompertz, B. P., O’Brien, P. T., Wynn, G. A., \& Rowlinson, A. 2013, MNRAS, 431,1745

Granot, J., \& Sari, R. 2002, ApJ, 568, 820

Granot, J., Königl, A., \& Piran, T. 2006, MNRAS, 370, 1946

Greiner, J., Bornemann, W., Clemens, C., et al. 2008, PASP, 120, 405

Hjorth, J., Sollerman, J., Gorosabel, J., et al. 2005a, ApJ, 630, L117

Hjorth, J., Watson, D., Fynbo, J. P. U., et al. 2005b, Nature, 437, 859

Kalberla, P. M. W., Burton, W. B., Hartmann, D., et al. 2005, A\&A, 440, 775

Kann, D. A., Klose, S., Zhang, B., et al. 2011, ApJ, 734, 96

Kann, D. A., Tanga, M., \& Greiner, J. 2015, GRB Coordinates Network, 17757, 1

Kaplan, D. L., Rowlinson, A., Bannister, K. W., et al. 2015, ApJ, 814, L25

Kouveliotou, C., Meegan, C. A., Fishman, G. J., et al. 1993, ApJ, 413, L101

Krühler, T., Küpcü Yoldaş, A., Greiner, J., et al. 2008, ApJ, 685, 376

Krühler, T., Schady, P., Greiner, J., et al. 2011, A\&A, 526, A153

Lattimer, J. M., \& Prakash, M. 2004, Science, 304, 536

Li, L., Liang, E.-W., Tang, Q.-W., et al. 2012, ApJ, 758, 27

Marshall, F. E., \& Beardmore, A. P. 2015, GRB Coordinates Network, 17751, 1

Mazets, E. P., Aptekar, R. L., Frederiks, D. D., et al. 2002, ArXiv e-prints [arXiv: astro-ph/0209219]

Mészáros, P., \& Rees, M. J. 1997, ApJ, 476, 232

Metzger, B. D., Quataert, E., \& Thompson, T. A. 2008, MNRAS, 385, 1455

Metzger, B. D., Margalit, B., Kasen, D., \& Quataert, E. 2015, MNRAS, 454, 3311

Nakar, E. 2007, Phys. Rep., 442, 166

Nicuesa Guelbenzu, A., Klose, S., Rossi, A., et al. 2011, A\&A, 531, L6

Norris, J. P., \& Bonnell, J. T. 2006, ApJ, 643, 266

Norris, J. P., Gehrels, N., \& Scargle, J. D. 2010, ApJ, 717, 411

Nysewander, M., Fruchter, A. S., \& Pe'er, A. 2009, ApJ, 701, 824

Panaitescu, A. 2005, MNRAS, 362, 921

Panaitescu, A., Mészáros, P., Gehrels, N., Burrows, D., \& Nousek, J. 2006, MNRAS, 366, 1357

Perley, D. A., \& McConnell, N. J. 2015, GRB Coordinates Network, 17745

Piran, T. 2004, Rev. Mod. Phys., 76, 1143

Planck Collaboration XVI. 2014, A\&A, 571, A16

Poole, T. S., Breeveld, A. A., Page, M. J., et al. 2008, MNRAS, 383, 627

Racusin, J. L., Liang, E. W., Burrows, D. N., et al. 2009, ApJ, 698, 43

Rea, N., Gullón, M., Pons, J. A., et al. 2015, ApJ, 813, 92

Roming, P. W. A., Kennedy, T. E., Mason, K. O., et al. 2005, Space Sci. Rev., 120,95

Roming, P. W. A., Vanden Berk, D., Pal'shin, V., et al. 2006, ApJ, 651, 985

Rowlinson, A., Gompertz, B. P., Dainotti, M., et al. 2014, MNRAS, 443, 1779

Sari, R., Piran, T., \& Narayan, R. 1998, ApJ, 497, L17

Schlafly, E. F., \& Finkbeiner, D. P. 2011, ApJ, 737, 103

Stratta, G., D’Avanzo, P., Piranomonte, S., et al. 2007, A\&A, 474, 827

Tanvir, N. R., Levan, A. J., Fruchter, A. S., et al. 2013, Nature, 500, 547

Tanvir, N. R., Levan, A. J., Fruchter, A. S., et al. 2015, GRB Coordinates Network, 18100, 1

Tody, D. 1993, in Astronomical Data Analysis Software and Systems II, eds.

R. J. Hanisch, R. J. V. Brissenden, \& J. Barnes, ASP Conf. Ser., 52, 173 van Eerten, H. 2014, MNRAS, 442, 3495

van Eerten, H. J. 2015, J. High Energy Astrophys., 7, 23

Wanderman, D., \& Piran, T. 2015, MNRAS, 448, 3026

Yi, I., \& Blackman, E. G. 1997, ApJ, 482, 383

Yoldaş, A. K., Krühler, T., Greiner, J., et al. 2008, in AIP Conf. Ser. 1000, eds. M. Galassi, D. Palmer, \& E. Fenimore, 227

Zhang, B., \& Mészáros, P. 2001, ApJ, 552, L35

Zhang, B., \& Mészáros, P. 2004, Int. J. Mod. Phys. A, 19, 2385

Zhang, B., Fan, Y. Z., Dyks, J., et al. 2006, ApJ, 642, 354

Zhang, B., Zhang, B.-B., Liang, E.-W., et al. 2007, ApJ, 655, L25 
F. Knust et al.: Long optical plateau in the afterglow of the short GRB 150424A with extended emission

\section{Appendix A: Additional tables}

Table A.1. All closure relations that are consistent with the data.

\begin{tabular}{|c|c|c|c|c|}
\hline CR & Scenario & Spectral regime & $p$ & $q$ \\
\hline \multicolumn{5}{|c|}{ X-ray before break } \\
\hline 1 & ISM, slow cooling & $v_{m}<v<v_{\mathrm{c}}$ & $2.82_{-0.13}^{+0.21}$ & $0.46 \pm 0.10$ \\
\hline 2 & ISM, slow cooling & $v>v_{\mathrm{c}}$ & $1.82_{-0.13}^{+0.21}$ & $0.71 \pm 0.17$ \\
\hline 4 & ISM, fast cooling & $v>v_{m}$ & $1.82_{-0.13}^{+0.21}$ & $0.71 \pm 0.17$ \\
\hline 5 & wind, slow cooling & $v_{m}<v<v_{\mathrm{c}}$ & $2.82_{-0.13}^{+0.21}$ & $-0.34 \pm 0.12$ \\
\hline 6 & wind, slow cooling & $v>v_{\mathrm{c}}$ & $1.82_{-0.13}^{+0.21}$ & $-1.39 \pm 0.08$ \\
\hline 8 & wind, fast cooling & $v>v_{m}$ & $1.82_{-0.13}^{+0.21}$ & $0.71 \pm 0.17$ \\
\hline 9 & uniform jet (spreading), slow cooling & $v_{m}<v<v_{\mathrm{c}}$ & $2.82_{-0.13}^{+0.21}$ & $0.32 \pm 0.05$ \\
\hline 10 & uniform jet (spreading), slow cooling & $v>v_{\mathrm{c}}$ & $1.82_{-0.13}^{+0.21}$ & $-2.08 \pm 0.32$ \\
\hline 11 & ISM, uniform jet (nonspreading), slow cooling & $v_{m}<v<v_{\mathrm{c}}$ & $2.82_{-0.13}^{+0.21}$ & $0.10 \pm 0.08$ \\
\hline 12 & ISM, Uniform jet (nonspreading), slow cooling & $v>v_{\mathrm{c}}$ & $1.82_{-0.13}^{+0.21}$ & $0.14 \pm 0.11$ \\
\hline 13 & wind, uniform jet (nonspreading), slow cooling & $v_{m}<v<v_{\mathrm{c}}$ & $2.82_{-0.13}^{+0.21}$ & $-0.22 \pm 0.08$ \\
\hline 14 & wind, uniform jet (nonspreading), slow cooling & $v>v_{\mathrm{c}}$ & $1.82_{-0.13}^{+0.21}$ & $0.46 \pm 0.10$ \\
\hline \multicolumn{5}{|c|}{$\mathrm{X}$-ray after break } \\
\hline 1 & ISM, slow cooling & $v_{m}<v<v_{\mathrm{c}}$ & $2.82_{-0.13}^{+0.21}$ & no energy injection needed \\
\hline 5 & wind, slow cooling & $v_{m}<v<v_{\mathrm{c}}$ & $2.82_{-0.13}^{+0.21}$ & no energy injection needed \\
\hline 6 & wind, slow cooling & $v>v_{\mathrm{c}}$ & $1.82_{-0.13}^{+0.21}$ & $-0.26 \pm 0.29$ \\
\hline 9 & uniform jet (spreading), slow cooling & $v_{m}<v<v_{\mathrm{c}}$ & $2.82_{-0.13}^{+0.21}$ & $0.87 \pm 0.14$ \\
\hline 11 & ISM, uniform jet (nonspreading), slow cooling & $v_{m}<v<v_{\mathrm{c}}$ & $2.82_{-0.13}^{+0.21}$ & $0.74 \pm 0.17$ \\
\hline 12 & ISM, uniform jet (nonspreading), slow cooling & $v>v_{\mathrm{c}}$ & $1.82_{-0.13}^{+0.21}$ & no energy injection needed \\
\hline 13 & wind, uniform jet (nonspreading), slow cooling & $v_{m}<v<v_{\mathrm{c}}$ & $2.82_{-0.13}^{+0.21}$ & $0.52 \pm 0.20$ \\
\hline 14 & wind, uniform jet (nonspreading), slow cooling & $v>v_{\mathrm{c}}$ & $1.82_{-0.13}^{+0.21}$ & no energy injection needed \\
\hline \multicolumn{5}{|c|}{ Optical before break } \\
\hline 1 & ISM, slow cooling & $v_{m}<v<v_{\mathrm{c}}$ & $1.82_{-0.13}^{+0.21}$ & $0.49 \pm 0.11$ \\
\hline 2 & ISM, slow cooling & $v>v_{\mathrm{c}}$ & $0.82_{-0.13}^{+0.21}$ & $0.84 \pm 0.21$ \\
\hline 3 & ISM, fast cooling & $v_{\mathrm{c}}<v<v_{m}$ & $n a_{-n a}^{+n a}$ & $0.74 \pm 0.08$ \\
\hline 4 & ISM, fast cooling & $v>v_{m}$ & $0.82_{-0.13}^{+0.21}$ & $0.84 \pm 0.21$ \\
\hline 5 & wind, slow cooling & $v_{m}<v<v_{\mathrm{c}}$ & $1.82_{-0.13}^{+0.21}$ & $-0.58 \pm 0.11$ \\
\hline 6 & wind, slow cooling & $v>v_{\mathrm{c}}$ & $0.82_{-0.13}^{+0.21}$ & $-2.00 \pm 0.01$ \\
\hline 7 & wind, fast cooling & $v_{\mathrm{c}}<v<v_{m}$ & $n a_{-n a}^{+n a}$ & $0.58 \pm 0.11$ \\
\hline 8 & wind, fast cooling & $v>v_{m}$ & $0.82_{-0.13}^{+0.21}$ & $0.84 \pm 0.21$ \\
\hline 9 & uniform jet (spreading), slow cooling & $v_{m}<v<v_{\mathrm{c}}$ & $1.82_{-0.13}^{+0.21}$ & $0.12 \pm 0.03$ \\
\hline 10 & uniform jet (spreading), slow cooling & $v>v_{\mathrm{c}}$ & $0.82_{-0.13}^{+0.21}$ & $-4.00 \pm 0.04$ \\
\hline 11 & ISM, uniform jet (nonspreading), slow cooling & $v_{m}<v<v_{\mathrm{c}}$ & $1.82_{-0.13}^{+0.21}$ & $0.06 \pm 0.07$ \\
\hline 12 & ISM, uniform jet (nonspreading), slow cooling & $v>v_{\mathrm{c}}$ & $0.82_{-0.13}^{+0.21}$ & $0.10 \pm 0.12$ \\
\hline 13 & wind, uniform jet (nonspreading), slow cooling & $v_{m}<v<v_{\mathrm{c}}$ & $1.82_{-0.13}^{+0.21}$ & $-0.34 \pm 0.07$ \\
\hline 14 & wind, uniform jet (nonspreading), slow cooling & $v>v_{\mathrm{c}}$ & $0.82_{-0.13}^{+0.21}$ & $0.49 \pm 0.11$ \\
\hline \multicolumn{5}{|c|}{ Optical after break } \\
\hline 6 & wind, slow cooling & $v>v_{\mathrm{c}}$ & $0.82_{-0.13}^{+0.21}$ & $0.02 \pm 0.16$ \\
\hline 11 & ISM, uniform jet (nonspreading), slow cooling & $v_{m}<v<v_{\mathrm{c}}$ & $1.82_{-0.13}^{+0.21}$ & no energy injection needed \\
\hline 13 & wind, uniform jet (nonspreading), slow cooling & $v_{m}<v<v_{\mathrm{c}}$ & $1.82_{-0.13}^{+0.21}$ & $0.84 \pm 0.13$ \\
\hline
\end{tabular}

Notes. The energy injection index $q$ is given where needed. 
Table A.2. AB magnitudes of comparison stars.

\begin{tabular}{l|rr|ccccccc}
\hline \hline$\#$ & RA & Dec & $g^{\prime}$ & $r^{\prime}$ & $i^{\prime}$ & $z^{\prime}$ & $J$ & $H$ & $K_{\mathrm{S}}$ \\
\hline I & 152.315 & -26.628 & $20.88 \pm 0.02$ & $19.96 \pm 0.01$ & $19.46 \pm 0.01$ & $19.19 \pm 0.01$ & $17.72 \pm 0.02$ & $17.05 \pm 0.03$ & $16.50 \pm 0.07$ \\
II & 152.297 & -26.628 & $22.40 \pm 0.06$ & $21.58 \pm 0.03$ & $21.26 \pm 0.04$ & $21.10 \pm 0.05$ & $19.82 \pm 0.15$ & $18.98 \pm 0.11$ & na \\
III & 152.299 & -26.635 & na & $21.10 \pm 0.01$ & $19.91 \pm 0.01$ & $19.39 \pm 0.01$ & $18.08 \pm 0.03$ & $17.43 \pm 0.03$ & $17.25 \pm 0.14$ \\
\hline
\end{tabular}

Notes. They do not include the systematical errors of the calibration: $g^{\prime}=0.03 \mathrm{mag}, r^{\prime}=0.03 \mathrm{mag}, i^{\prime}=0.04 \mathrm{mag}, z^{\prime}=0.04 \mathrm{mag}, J=0.05 \mathrm{mag}$, $H=0.05 \mathrm{mag}$, and $K=0.07 \mathrm{mag}$.

Table A.3. Photometry of GRB 150424A.

\begin{tabular}{|c|c|c|c|c|c|}
\hline Time & Time error & $\mathrm{AB}$ mag & mag error & Band & Instrument \\
\hline 57903 & 2312 & 21.76 & 0.04 & $g^{\prime}$ & GROND \\
\hline 62645 & 2325 & 21.90 & 0.03 & $g^{\prime}$ & GROND \\
\hline 67277 & 2220 & 21.99 & 0.04 & $g^{\prime}$ & GROND \\
\hline 156123 & 4528 & 23.32 & 0.10 & $g^{\prime}$ & GROND \\
\hline 238900 & 5428 & 23.6 & UL & $g^{\prime}$ & GROND \\
\hline 323218 & 2930 & 24.5 & UL & $g^{\prime}$ & GROND \\
\hline 411767 & 5450 & 24.9 & UL & $g^{\prime}$ & GROND \\
\hline 582978 & 3189 & 23.4 & UL & $g^{\prime}$ & GROND \\
\hline 670665 & 5245 & 23.9 & UL & $g^{\prime}$ & GROND \\
\hline 842737 & 4961 & 24.2 & UL & $g^{\prime}$ & GROND \\
\hline 57903 & 2312 & 21.55 & 0.03 & $r^{\prime}$ & GROND \\
\hline 62645 & 2325 & 21.60 & 0.03 & $r^{\prime}$ & GROND \\
\hline 67277 & 2220 & 21.80 & 0.03 & $r^{\prime}$ & GROND \\
\hline 156582 & 4986 & 23.08 & 0.07 & $r^{\prime}$ & GROND \\
\hline 238900 & 5428 & 24.4 & UL & $r^{\prime}$ & GROND \\
\hline 323218 & 2930 & 23.92 & 0.22 & $r^{\prime}$ & GROND \\
\hline 411767 & 5450 & 25.0 & UL & $r^{\prime}$ & GROND \\
\hline 582978 & 3189 & 23.9 & UL & $r^{\prime}$ & GROND \\
\hline 670891 & 5471 & 24.1 & UL & $r^{\prime}$ & GROND \\
\hline 842962 & 5186 & 24.5 & UL & $r^{\prime}$ & GROND \\
\hline 57903 & 2312 & 21.45 & 0.05 & $i^{\prime}$ & GROND \\
\hline 62645 & 2325 & 21.44 & 0.04 & $i^{\prime}$ & GROND \\
\hline 67374 & 2316 & 21.58 & 0.05 & $i^{\prime}$ & GROND \\
\hline 156582 & 4986 & 23.03 & 0.13 & $i^{\prime}$ & GROND \\
\hline 238900 & 5428 & 23.9 & UL & $i^{\prime}$ & GROND \\
\hline 323218 & 2930 & 24.1 & UL & $i^{\prime}$ & GROND \\
\hline 411767 & 5450 & 24.6 & UL & $i^{\prime}$ & GROND \\
\hline 582978 & 3189 & 23.6 & UL & $i^{\prime}$ & GROND \\
\hline 670891 & 5471 & 23.8 & UL & $i^{\prime}$ & GROND \\
\hline 842962 & 5186 & 24.3 & UL & $i^{\prime}$ & GROND \\
\hline 57903 & 2312 & 21.29 & 0.06 & $z^{\prime}$ & GROND \\
\hline 62645 & 2325 & 21.40 & 0.05 & $z^{\prime}$ & GROND \\
\hline 67374 & 2316 & 21.52 & 0.06 & $z^{\prime}$ & GROND \\
\hline 156355 & 4760 & 22.62 & 0.12 & $z^{\prime}$ & GROND \\
\hline 238900 & 5428 & 23.6 & UL & $z^{\prime}$ & GROND \\
\hline 323218 & 2930 & 23.8 & UL & $z^{\prime}$ & GROND \\
\hline 411767 & 5450 & 24.4 & UL & $z^{\prime}$ & GROND \\
\hline 582978 & 3189 & 23.3 & $\mathrm{UL}$ & $z^{\prime}$ & GROND \\
\hline 670891 & 5471 & 23.0 & UL & $z^{\prime}$ & GROND \\
\hline 842962 & 5186 & 24.0 & UL & $z^{\prime}$ & GROND \\
\hline 57929 & 2338 & 20.98 & 0.19 & $J$ & GROND \\
\hline 62670 & 2351 & 21.22 & 0.19 & $J$ & GROND \\
\hline 67399 & 2343 & 21.38 & 0.23 & $J$ & GROND \\
\hline 156606 & 5011 & 22.1 & UL & $J$ & GROND \\
\hline 238924 & 5452 & 22.0 & UL & $J$ & GROND \\
\hline 323331 & 3043 & 21.9 & UL & $J$ & GROND \\
\hline 411791 & 5475 & 22.4 & UL & $J$ & GROND \\
\hline
\end{tabular}

Notes. Upper limits are flagged with "UL". 
F. Knust et al.: Long optical plateau in the afterglow of the short GRB 150424A with extended emission

Table A.3. continued.

\begin{tabular}{|c|c|c|c|c|c|}
\hline Time & Time error & $\mathrm{AB}$ mag & mag error & Band & Instrument \\
\hline 670915 & 5494 & 22.1 & UL & $J$ & GROND \\
\hline 842987 & 5211 & 22.4 & UL & $J$ & GROND \\
\hline 156606 & 5011 & 21.6 & UL & $H$ & GROND \\
\hline 238924 & 5452 & 21.6 & UL & $H$ & GROND \\
\hline 323331 & 3043 & 21.5 & UL & $H$ & GROND \\
\hline 411791 & 5475 & 22.0 & UL & $H$ & GROND \\
\hline 670915 & 5494 & 21.6 & UL & $H$ & GROND \\
\hline 842987 & 5211 & 21.7 & UL & $H$ & GROND \\
\hline 156834 & 5239 & 20.5 & UL & $K_{\mathrm{S}}$ & GROND \\
\hline 238924 & 5452 & 20.0 & UL & $K_{\mathrm{S}}$ & GROND \\
\hline 323242 & 2954 & 19.9 & UL & $K_{\mathrm{S}}$ & GROND \\
\hline 411791 & 5475 & 20.4 & UL & $K_{\mathrm{S}}$ & GROND \\
\hline 670915 & 5494 & 20.5 & UL & $K_{\mathrm{S}}$ & GROND \\
\hline 843441 & 5665 & 20.9 & UL & $K_{\mathrm{S}}$ & GROND \\
\hline 436 & 123 & 21.3 & UL & $u$ & UVOT \\
\hline 2887 & 108 & 20.8 & $\mathrm{UL}$ & $u$ & UVOT \\
\hline 6396 & 98 & 20.56 & 0.23 & $u$ & UVOT \\
\hline 3404 & 329 & 20.91 & 0.17 & $u$ & UVOT \\
\hline 45591 & 433 & 21.3 & UL & $u$ & UVOT \\
\hline 99360 & 305 & 21.0 & UL & $u$ & UVOT \\
\hline 185608 & 300 & 20.8 & UL & $u$ & UVOT \\
\hline 795276 & 284 & 21.3 & UL & $u$ & UVOT \\
\hline 1394595 & 4354 & 22.1 & UL & $u$ & UVOT \\
\hline 664 & 19 & 18.9 & UL & $b$ & UVOT \\
\hline 5884 & 197 & 20.42 & 0.25 & $b$ & UVOT \\
\hline 3634 & 216 & 20.9 & UL & $b$ & UVOT \\
\hline 16166 & 443 & 20.5 & $\mathrm{UL}$ & $b$ & UVOT \\
\hline 16166 & 443 & 21.0 & UL & $b$ & UVOT \\
\hline 129408 & 590 & 20.7 & UL & $b$ & UVOT \\
\hline 82 & 5 & 17.5 & UL & $v$ & UVOT \\
\hline 738 & 19 & 18.0 & UL & $v$ & UVOT \\
\hline 4347 & 98 & 19.1 & UL & $v$ & UVOT \\
\hline 5782 & 98 & 19.6 & UL & $v$ & UVOT \\
\hline 3261 & 216 & 19.8 & UL & $v$ & UVOT \\
\hline 12078 & 295 & 19.9 & UL & $v$ & UVOT \\
\hline 20295 & 589 & 20.1 & UL & $v$ & UVOT \\
\hline 34741 & 442 & 19.9 & UL & $v$ & UVOT \\
\hline 160892 & 588 & 19.9 & UL & $v$ & UVOT \\
\hline 2773 & 108 & 20.9 & UL & $u v w 1$ & UVOT \\
\hline 17934 & 329 & 21.27 & 0.19 & $u v w 1$ & UVOT \\
\hline 42244 & 560 & 22.2 & UL & $u v w 1$ & UVOT \\
\hline 92657 & 358 & 22.1 & $\mathrm{UL}$ & $u v w 1$ & UVOT \\
\hline 177963 & 461 & 21.8 & UL & $u v w 1$ & UVOT \\
\hline 746677 & 1086 & 22.4 & UL & $u v w 1$ & UVOT \\
\hline 1310529 & 5447 & 22.6 & UL & $u v w 1$ & UVOT \\
\hline 714 & 19 & 19.7 & UL & $u v w 2$ & UVOT \\
\hline 3349 & 106 & 21.2 & UL & $u v w 2$ & UVOT \\
\hline 3146 & 126 & 21.9 & UL & $u v w 2$ & UVOT \\
\hline 11320 & 443 & 21.65 & 0.17 & $u v w 2$ & UVOT \\
\hline 29251 & 1618 & 21.89 & 0.10 & $u v w 2$ & UVOT \\
\hline 129298 & 554 & 22.6 & UL & $u v w 2$ & UVOT \\
\hline 215616 & 640 & 22.5 & $\mathrm{UL}$ & $u v w 2$ & UVOT \\
\hline 431143 & 3532 & 23.1 & UL & $u v w 2$ & UVOT \\
\hline 1295417 & 6031 & 23.2 & UL & $u v w 2$ & UVOT \\
\hline 762 & 19 & 19.1 & UL & uvm2 & UVOT \\
\hline 5269 & 197 & 21.2 & UL & uvm2 & UVOT \\
\hline 3376 & 206 & 21.7 & UL & uvm2 & UVOT \\
\hline 72406 & 470 & 21.9 & UL & uvm2 & UVOT \\
\hline
\end{tabular}


A\&A 607, A84 (2017)

Table A.3. continued.

\begin{tabular}{|c|c|c|c|c|c|}
\hline Time & Time error & $\mathrm{AB}$ mag & mag error & Band & Instrument \\
\hline 173186 & 1105 & 22.3 & UL & uvm2 & UVOT \\
\hline 516450 & 2747 & 22.5 & UL & uvm2 & UVOT \\
\hline 1224527 & 5590 & 22.4 & UL & uvm 2 & UVOT \\
\hline 174 & 74 & 21.30 & 0.23 & white & UVOT \\
\hline 601 & 10 & 20.7 & UL & white & UVOT \\
\hline 890 & 83 & 20.94 & 0.16 & white & UVOT \\
\hline 5371 & 98 & 20.96 & 0.15 & white & UVOT \\
\hline 8481 & 418 & 20.93 & 0.08 & white & UVOT \\
\hline 10563 & 295 & 20.87 & 0.09 & white & UVOT \\
\hline 16926 & 295 & 21.09 & 0.10 & white & UVOT \\
\hline 32126 & 1053 & 21.02 & 0.06 & white & UVOT \\
\hline 292642 & 1707 & 22.8 & UL & white & UVOT \\
\hline 303903 & 1184 & 22.7 & UL & white & UVOT \\
\hline 314852 & 534 & 22.2 & UL & white & UVOT \\
\hline 335542 & 427 & 22.3 & UL & white & UVOT \\
\hline 358922 & 1178 & 22.6 & UL & white & UVOT \\
\hline 576743 & 2047 & 24.96 & 0.25 & $F 125 W$ & HST \\
\hline 800368 & 2197 & 25.53 & 0.26 & $F 125 W$ & HST \\
\hline 1203117 & 811 & 26.01 & 0.26 & $F 125 W$ & HST \\
\hline 579662 & 811 & 24.73 & 0.25 & $F 160 W$ & HST \\
\hline 580609 & 75 & 24.55 & 0.25 & $F 160 W$ & HST \\
\hline 803437 & 811 & 25.26 & 0.25 & $F 160 W$ & HST \\
\hline 1206264 & 2274 & 25.57 & 0.26 & $F 160 W$ & HST \\
\hline 573431 & 1092 & 26.03 & 0.26 & $F 606 W$ & HST \\
\hline 796906 & 1092 & 26.25 & 0.26 & $F 606 W$ & HST \\
\hline 5604 & 180 & 20.77 & 0.03 & $g^{\prime}$ & LRIS \\
\hline 5616 & 180 & 20.66 & 0.04 & $R_{\mathrm{c}}$ & LRIS \\
\hline
\end{tabular}

